\title{
Colloidal layers in magnetic fields and under shear flow
}

\author{
H Löwen ${ }^{1}$, R Messina ${ }^{1}$, N Hoffmann ${ }^{1}$, C N Likos ${ }^{1}$, C Eisenmann ${ }^{2}$,

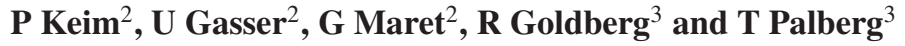 \\ ${ }^{1}$ Institut für Theoretische Physik II, Heinrich-Heine-Universität Düsseldorf, Universitätsstraße 1, \\ D-40225 Düsseldorf, Germany \\ ${ }^{2}$ Fachbereich Physik, Universität Konstanz, Postfach 5560, 78457 Konstanz, Germany \\ ${ }^{3}$ Institut für Physik, Universität Mainz, KOMET 336 Staudinger Weg 7, D-55128 Mainz, \\ Germany
}

E-mail: hlowen@thphy.uni-duesseldorf.de

Received 16 September 2005

Published 28 October 2005

Online at stacks.iop.org/JPhysCM/17/S3379

\begin{abstract}
The behaviour of colloidal mono- and bilayers in external magnetic fields and under shear is discussed and recent progress is summarized. Superparamagnetic colloidal particles form monolayers when they are confined to a air-water interface in a hanging water droplet. An external magnetic field allows us to tune the strength of the mutual dipole-dipole interaction between the colloids and the anisotropy of the interaction can be controlled by the tilt angle of the magnetic field relative to the surface normal of the air-water interface. For sufficiently large magnetic field strength crystalline monolayers are found. The role of fluctuations in these two-dimensional crystals is discussed. Furthermore, clustering phenomena in binary mixtures of superparamagnetic particles forming fluid monolayers are predicted. Finally, we address sheared colloidal bilayers and find that the orientation of confined colloidal crystals can be tailored by a previously applied shear direction.
\end{abstract}

(Some figures in this article are in colour only in the electronic version)

\section{Introduction}

A strongly confined system possesses properties that widely differ from those in the bulk. The most extreme situation is to confine a three-dimensional system down to a few molecular layers, e.g., by adsorbing the material on an attractive substrate. Mesoscopic colloidal particles are excellent model systems to study confinement effects on a scale of an interparticle distance. By using external fields [1], the colloidal system can be placed into controlled equilibrium and non-equilibrium situations. 


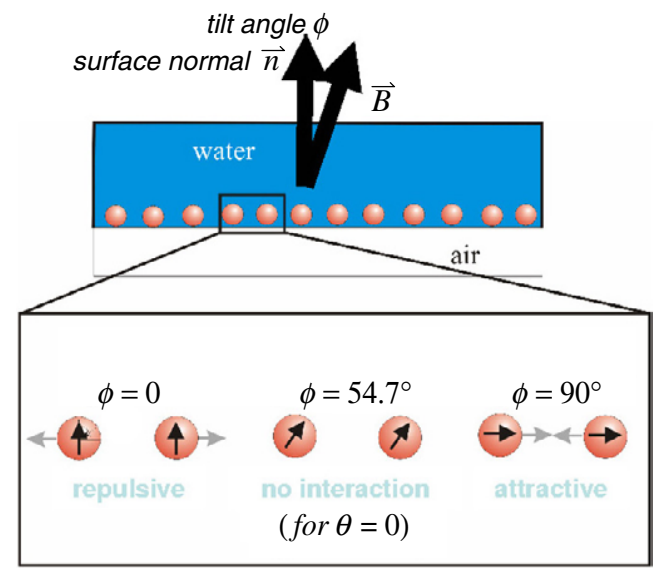

Figure 1. Schematic view of the set-up: superparamagnetic colloidal particles at an air-water interface in an external magnetic field $\vec{B}$ with a tilt angle $\phi$ inducing dipole moments in the particles. The induced dipole moments and their mutual interaction are also shown for different tilt angles $\phi$ of the external magnetic field.

In this paper we review some recent developments in this field and also describe some new original results. In particular, we address superparamagnetic colloids which are confined to a two-dimensional water-air interface of a pendent droplet. These provide excellent model systems for strictly two-dimensional strongly interacting classical many-body systems and phase transitions like melting, freezing and vitrification can be studied in those systems in real space. Here we review the anisotropic melting process and present the possibility of clustered fluid in binary mixtures of magnetic colloids.

Another possibility to confine colloidal dispersions is to squeeze them between two parallel glass plates. This can be done down to one or two colloidal layers and again the freezing process can be studied in real space in these low-dimensional systems. Further external fields such as shear can be applied to push the system out of equilibrium in a controlled way. This will have a drastic influence on the equilibrium phase transition and can induce novel effects which are not seen in equilibrium. As an example, we show here that shear flow can be used to tune the orientation of a confined colloidal crystal. Shear melts confined crystals and after cessation of shear the crystal preferentially picks its orientation according to the previous shear flow. This effect can in principle be exploited in constructing optical switching elements.

The paper is organized as follows: in section 2, we summarize recent progress obtained in studying monolayers of superparamagnetic colloids. We then discuss confined charged suspensions in equilibrium and under shear in section 3 and we conclude in section 4.

\section{Monolayers of superparamagnetic colloidal particles in external magnetic fields}

Spherical colloidal particles which are superparamagnetic due to $\mathrm{Fe}_{2} \mathrm{O}_{3}$ doping are confined in a pendent water droplet. Gravity pulls them downwards until they reach the water-air interface. The high tension of the free water-air interface, on the other hand, does not allow a penetration of the colloidal particles. Hence they are confined to the interface and perpendicular fluctuations are small. The interface of the water droplet is planar on the scale of the interparticle separation. Therefore, this system is an excellent realization of a two-dimensional monolayer of particles. If an external magnetic field $\vec{B}$ is applied (see figure 1 for the schematic set-up), it 


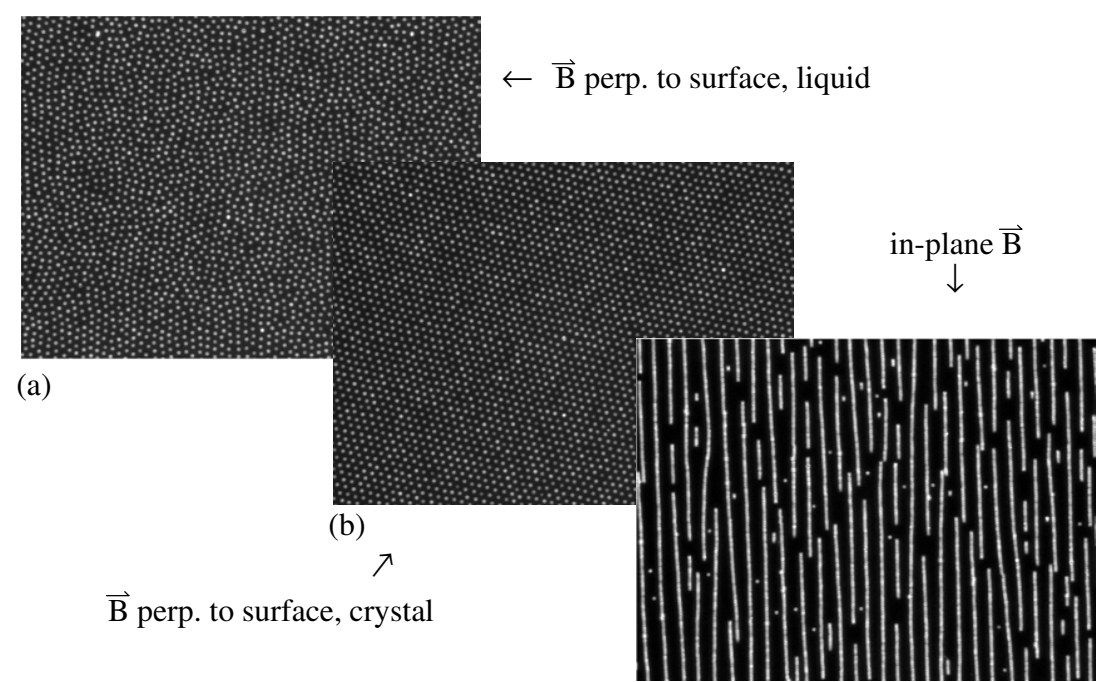

(c)

Figure 2. Particle configurations for different fields: (a) $\phi=0$, liquid phase; (b) $\phi=0$, crystal phase; (c) $\phi=90^{\circ}$, dipolar chain phase.

induces a magnetic dipole moment $\vec{m}=\chi \vec{B}$ in each of the particles such that the particles are interacting dipoles. Here $\chi$ denotes the magnetic susceptibility. The resulting dipole-dipole pair interaction energy between the particles reads together with a stabilizing hard-core part $V_{\mathrm{hc}}$ as

$$
V(\vec{r})=V_{\mathrm{hc}}+\frac{m^{2}}{2} \frac{1}{r^{3}}\left(1-3 \cos ^{2} \phi \cos ^{2} \Theta\right)
$$

where $r$ is the distance between two particles, $\phi$ is the tilt angle of the external magnetic field with respect to the surface normal $\vec{n}$ (see figure 1) and $\Theta$ is the angle between the twodimensional separation vector $\vec{r}$ and the projection $\vec{B}_{\|}$of the magnetic field down to the airwater interface. According to (1), the form of the interaction can be tuned by the external field. The field amplitude $B$ sets the interaction strength and is therefore equivalent to an inverse system temperature while the tilt angle $\phi$ governs the relative importance of the attraction. In fact, for $\Theta=0$, as shown in figure 1, there is pure repulsion for perpendicular magnetic fields $(\phi=0)$ corresponding to parallel dipole moments. Dipole attraction is obtained for the opposite extreme case $\phi=90^{\circ}$ while at an intermediate value $\phi=54.7^{\circ}$ there is no interaction at all.

Particle configurations as obtained by video microscopy for different external magnetic fields are shown in figure 2. For a perpendicular field, there is either a two-dimensional liquid or a two-dimensional triangular (or hexagonal) crystal, while for perpendicular fields the mutual attraction leads to strong chain formation of two-dimensional dipolar spheres.

Using these systems for zero tilt angle, the harmonic lattice behaviour ("phonon spectra') was obtained and quantitative agreement with harmonic lattice calculations based on the dipole-dipole potential (1) $\propto 1 / r^{3}$ was found [2]. Furthermore, the two-dimensional Kosterlitz-Thouless-Nelson-Halperin-Young (KTHNY) melting scenario has been confirmed in detail. In particular, it has been shown recently [3] that Young's elastic modulus equals $16 \pi$ at the melting transition from a triangular crystal to a hexatic phase. This is a direct experimental verification of the predictions of KTHNY theory. 


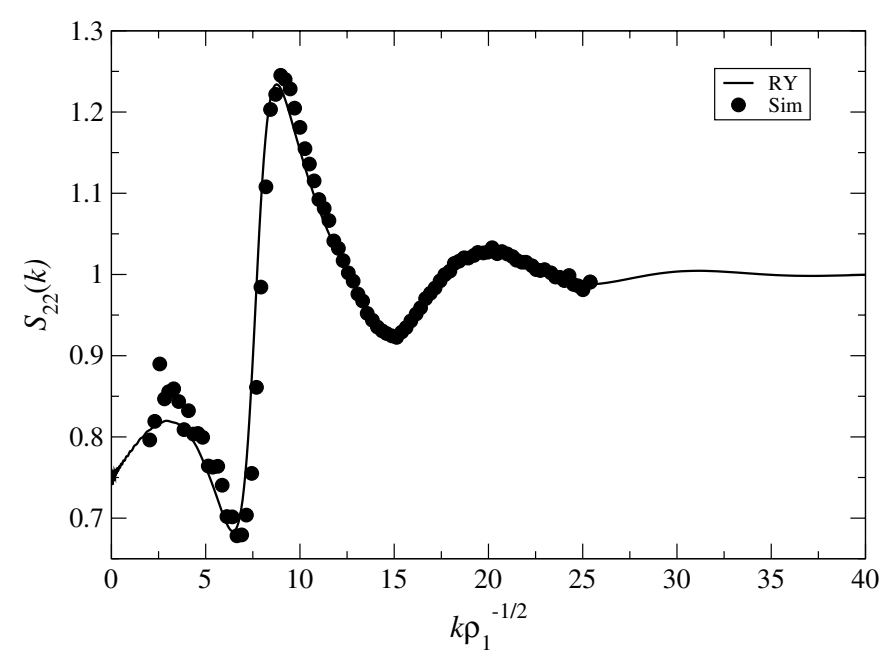

Figure 3. Partial structure factor $S_{22}(k)$ of the correlations between the small particles in a twodimensional binary mixture of magnetic colloids. Solid line: liquid integral equation theory using the Rogers-Young (RY) closure. Filled circles: computer simulation data. Apart from the principal peak at $k \rho_{1}^{-1 / 2} \approx 10$, there is another cluster peak at smaller wavevectors $k \rho_{1}^{-1 / 2} \approx 4$ (with $\rho_{1}$ denoting the number density of the big particles). The other parameters of the mixtures are $\Gamma_{11}=4.0, \rho_{2} / \rho_{1}=0.5$ and $\chi_{2} / \chi_{1}=0.5$.

For non-zero tilt angles $(\phi \geqslant 0)$ the full phase diagram for arbitrary $\phi$ and colloidal number density has been calculated at zero temperature by using minimization of lattice sums [4]. It includes a rich variety of different stable two-dimensional crystal lattices with rectangular, rectangular-centred, oblique, rhombic and chain-like oblique phases and several first-order and second-order phase transitions among them. For small number densities and small tilt angles, where the dependence on the physical hard core is irrelevant, a detailed comparison between theory and experiment of the lattice anisotropy in the rectangular-centred crystal has revealed perfect agreement [5]. Furthermore, the anisotropic relative mean-square displacements in the solid have been examined as a function of the tilt angle $\phi$ in both harmonic lattice calculations and experiments, and very good agreement was found [6].

The melting of such anisotropic crystals was also explored in recent experiments [7], and the role of anisotropic defect formation was observed. For large anisotropies $\left(\phi \geqslant 23.7^{\circ}\right)$, the anisotropic crystals melt into a smectic (or columnar) phase, intermediate between solid and liquid. This phase exhibits liquid-like behaviour parallel to the direction of $\vec{B}_{\|}$, but long-range solid-like order perpendicular to this direction.

Further, we address the case of binary mixtures of superparamagnetic particles with two different susceptibilities $\chi_{1}$ and $\chi_{2}$. Of particular interest here is the ability to tune, via control of the susceptibility asymmetry, the effective non-additivity of the mixture. Such mixtures have been considered for vitrification [8] and for dynamical properties [9], but the fluid phase behaviour is not yet thoroughly understood. A theoretical calculation based on a dipole-dipole interaction for zero tilt angle $\phi$ and employing the Rogers-Young liquid integral equation closures [10] predicts an interesting small- $k$ peak in the partial structure factor $S_{22}(k)$ of the small particles; see figure 3 .

The small- $k$ peak is confirmed by Brownian dynamics computer simulations of the mixture. It signals the emergence of a new length scale in the system. Simulation snapshots, as presented in figure 4, reveal a clustering of the small particles with an intercluster spacing 

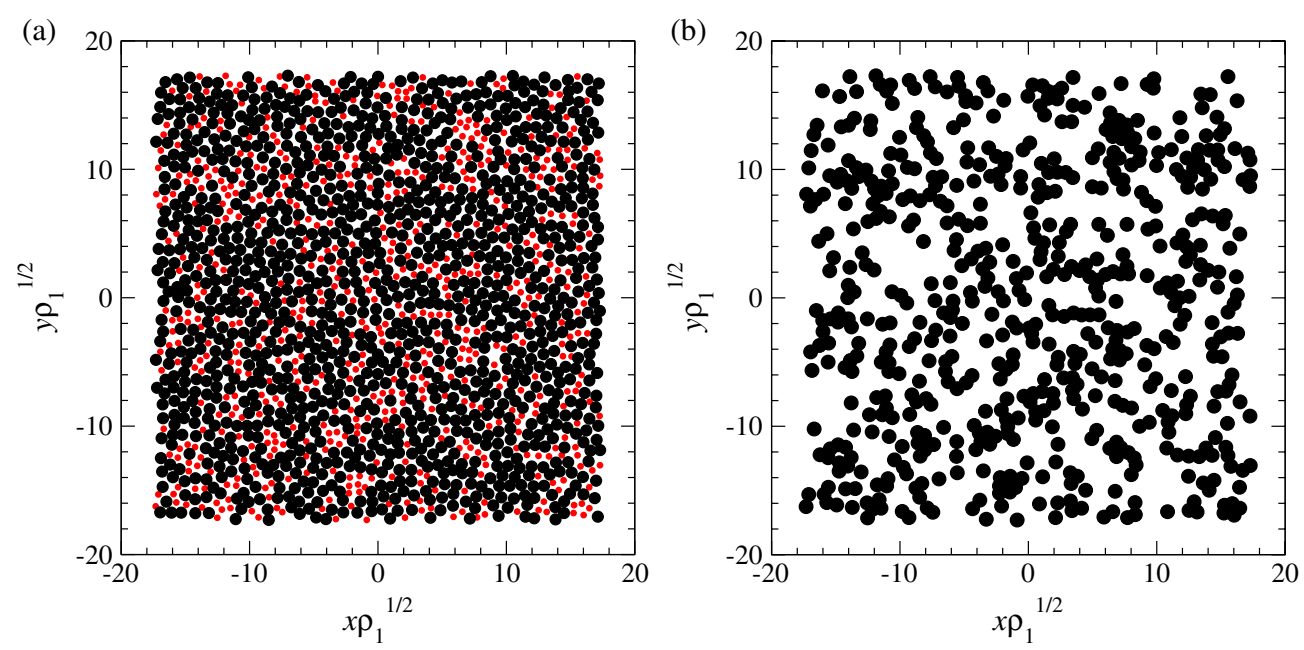

Figure 4. Computer simulation snapshots corresponding to the state point examined in figure 3 . (a) Both big and small particles are shown. (b) Only the small particles are shown (represented by larger circles than in (a)) in order to reveal the void structure.

that corresponds to the small- $k$ peak in $S_{22}(k)$. Details of the cluster structure should be characterized further by morphological measures such as the Euler characteristic. This is a topic for future consideration [10].

Clustering in binary colloidal systems has been observed before. It is therefore interesting to compare the present results to two other model cases. First, at elevated densities and larger fields the 2D magnetic fluids undergo a phase transition into a 2D solid. Except for certain well defined susceptibility ratios the solid does not display a long ranged binary order. Instead, local clusters of high symmetry emerge (so-called elementary triangle clusters), which due to geometrical frustration may not arrange in a plane-filling manner [11]. This kind of amorphous solid is the 2D counterpart of a 3D Bernal glass [12].

Second, clustering may also be observed in other purely repulsive mixtures, e.g. in mixtures of hard or charged spheres with neutral polymer or polyelectrolyte [13-15]. In figure 5 we show the first observation of a fluid of stable large sphere clusters appearing in a binary charged sphere mixture with sedimented large spheres. Light scattering shows that the small particles take a homogeneous fluid structure. One observes stable finite size clusters of five to ten particles, which display an irregular shape and are rather mobile. The inter-cluster spacing is about 10-15 large particle diameters. This fluid-like structure is caused by the mutual repulsion between the clusters due to the accumulated charge. This stabilization mechanism specific to charged systems with their long ranged repulsion has been compared to the fluid-drop model of the atomic nucleus [16]. It is different to the phase-separation-like gel formation observed in the hard-sphere/polymer case.

\section{Sheared bilayers of confined charged suspensions}

Another way to drastically confine suspensions is to squeeze them between two parallel glass plates. Again, the particle configurations can then be watched in real space by video microscopy [17-19]. Typically, charged colloidal suspensions and highly charged plates are used. The strength of the mutual repulsive interaction can be tuned by added salt. A Yukawa 


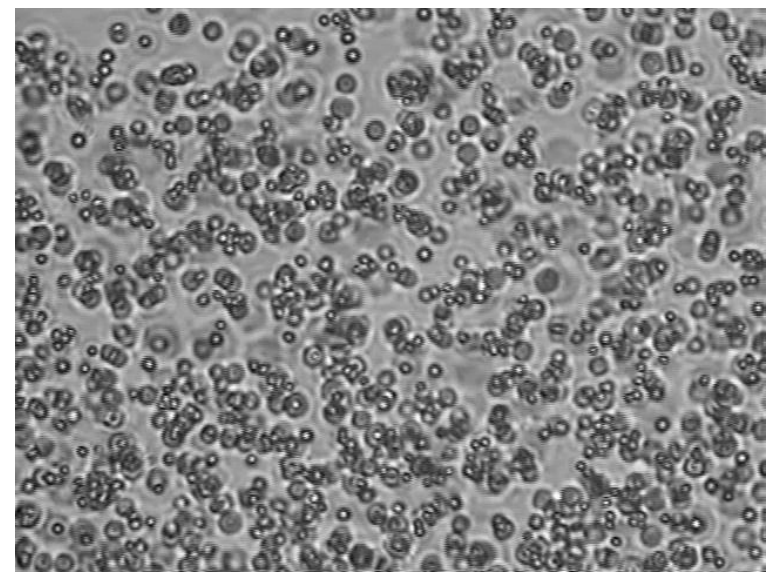

Figure 5. Microscopic image of a 2D charged sphere binary mixture. The aqueous suspension contains a mixture of sedimenting large spheres (diameter $1.2 \mu \mathrm{m}$ ) with small spheres (diameter $68 \mathrm{~nm})$ at a number ratio of about $n_{S} / n_{L} \approx 10^{5}$ and under low salt conditions $(c=6 \times$ $\left.10^{-5} \mathrm{~mol}^{-1}\right)$. Here only the large particle clusters are visible in microscopy. One observes finite size clusters of five to ten particles, which display an irregular shape and are rather mobile. They show considerable shape fluctuations, and also number fluctuations appear on a time scale of several seconds. The average particle number per cluster, however, is constant and thus documents the equilibrium character of the situation. The inter-cluster spacing is quite large, with a preferred distance of about 10-15 large particle diameters.

pair potential between the colloidal particles involving an inverse screening length $\kappa$ and an exponentially decaying wall-particle potential involving the same $\kappa$ are an appropriate theoretical model for charged suspensions.

In equilibrium there is a wealth of different crystalline phases, even if the number of layers is fixed. For bilayers, the whole phase diagram has been calculated for various inverse screening lengths and different particle densities at zero temperature [20]. Stable phases are two nested rectangular, quadratic or triangular lattices or rhombic sheets with two different relative displacements. Recent experiments [21] have confirmed the theoretical phase diagram.

If one plate is sheared relative to the other one, the system is placed in a nonequilibrium situation. If the starting equilibrium configuration is a crystalline bilayer, the following scenario is found via Brownian dynamics computer simulation of a confined Yukawa system [24]: at small shear rates the elastic behaviour of the solid is tested. For increasing shear rates, there is a yield shear rate above which the system starts to flow. Increasing the shear rate further results in a shear-molten solid. For very high shear rates the particles align along the shear direction, so that long-range order comes back, leading to a re-entrance of crystallization. Qualitatively, the same scenario has been seen in the bulk by experiments and simulations $[22,23]$.

Here we show-using similar Brownian dynamics computer simulations of a confined Yukawa system as in [24] - that the orientation of a confined crystal can be tuned at wish by a previously applied shear direction. In order to demonstrate this, three typical particle configurations are shown in figure 6 . The starting configuration is a nested square bilayer, see figure 6(a). The nearest neighbour direction is oriented at $45^{\circ}$ to the axes of the simulation box. Then shear is applied in the direction of the $x$-axis of the simulation box. The solid is strongly affected by shear and a steady-state configuration is shown in figure 6(b). Then shear is released and the system relaxes back to the nested two-square crystal lattice. The previous preordering according to the shear direction then selects a crystal orientation where the nearest 

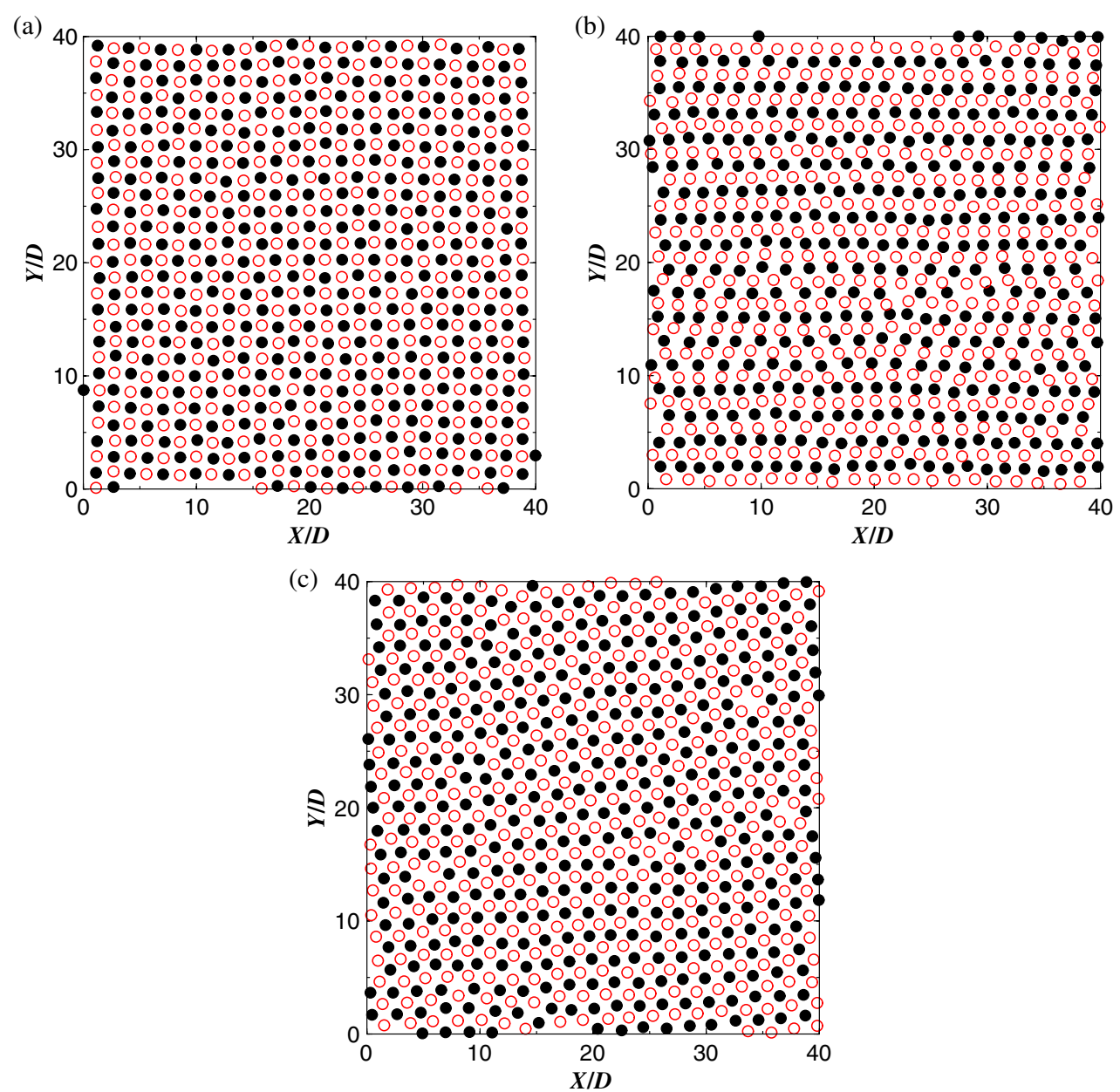

Figure 6. Simulation snapshots at different regimes. (a) The starting equilibrium (i.e., $\dot{\gamma}=0$ ) configuration. (b) Steady state at $\dot{\gamma}=200 / \tau$ where the shear is applied in the $X$-direction. (c) Relaxed state after cessation of shear. The filled (open) circles represent particles belonging to the upper (lower) layer. The reduced screening strength is here $\kappa D=2.5$ and the reduced density is $\rho D^{2} / 2=0.24$ where $D$ represents the layer-layer separation. $\tau=D^{2} / D_{0}$ is the simulation timescale, with $D_{0}$ denoting the free diffusion constant.

neighbour separation lies parallel to the shear direction. This effect can be used to tailor crystal orientation at will, which is important for the fabrication of optical switching elements.

\section{Conclusions}

Colloidal suspensions in strong confinement represent excellent realizations of few-layer systems. Phase transitions such as freezing, melting and vitrification can be studied in realspace and lead to various phenomena, such as to the anisotropic KTHNY scenario and various solid-to-solid transformations. Shear can destroy crystalline order but generates new order as well. In particular, by applying shear, the orientation of a crystal can be tuned at wish.

Future work will focus on different directions: first, one may go from mono- and bilayers to systems composed of few layers. The shear behaviour of hard-sphere colloids was recently 
investigated in real space $[25,26]$. Second, one may go from one-component systems to binary mixtures. The crystallization of binary magnetic suspensions and of binary charged suspensions has not yet been investigated in detail. Finally, one may study situations with spatially inhomogeneous and time-dependent external fields (both magnetic and shear), where a wealth of new phenomena is expected. For example, by a combination of shear and confinement it might be possible to observe crystallized clusters in binary mixtures of magnetic particles.

\section{Acknowledgments}

This work was supported by the German Science Foundation (DFG) via the Collaborative Research Centre SFB TR6 (Physics of colloidal dispersions in external fields), project sections C2, C3 and D1.

\section{References}

[1] Löwen H 2001 J. Phys.: Condens. Matter 13 R415

[2] Keim P, Maret G, Herz U and von Grünberg H H 2004 Phys. Rev. Lett. 92215504

[3] von Grünberg H H, Keim P, Zahn K and Maret G 2004 Phys. Rev. Lett. 93255703

[4] Froltsov V A, Blaak R, Likos C N and Löwen H 2003 Phys. Rev. E 68061406

[5] Eisenmann C, Keim P, Gasser U and Maret G 2004 J. Phys.: Condens. Matter 16 S4095

[6] Froltsov V, Likos C N, Löwen H, Eisenmann C, Gasser U, Keim P and Maret G 2005 Phys. Rev. E 71031404

[7] Eisenmann C, Gasser U, Keim P and Maret G 2004 Phys. Rev. Lett. 93105702

[8] König H, Hund R, Zahn K and Maret G 2004 Experimental realisation of a colloidal glass former in 2D, submitted

[9] Kollmann M, Hund R, Rinn B, Nägele G, Zahn K, König H, Maret G, Klein R and Dhont J K G 2002 Europhys. Lett. $\mathbf{5 8} 919$

[10] Hoffmann N, Likos C N and Löwen H 2005 Microphase structuring in two-dimensional magnetic colloid mixtures, at press

[11] König H 2005 Europhys. Lett. 71838

[12] Bernal D 1960 Nature $\mathbf{1 8 8} 908$ Bernal D 1960 Nature 18568

[13] Eckert T and Bartsch E 2002 Phys. Rev. Lett. 89125701 Eckert T and Bartsch E 2004 J. Phys.: Condens. Matter 16 S4937

[14] Zaccarelli E, Sciortino F, Tartaglia P, Foffi G, McCullagh G D, Lawlor A and Dawson K A 2002 Physica A $314539-47$

[15] Sedgwick H, Egelhaaf S U and Poon W C K 2004 J. Phys.: Condens. Matter 164913

[16] Groenewoldt J and Kegel W 2001 J. Phys. Chem. B 10511702

[17] Biehl R and Palberg T 2004 Rev. Sci. Instrum. 75906

[18] Biehl R and Palberg T 2003 Proc. R. Chem. Soc. Faraday Discuss. 7123133

[19] Biehl R and Palberg T 2004 Europhys. Lett. 66291

[20] Messina R and Löwen H 2003 Phys. Rev. Lett. E 91146101

[21] Barreira Fontecha A, König H, Palberg T, Messina R and Löwen H 2005 J. Phys.: Condens. Matter 17 S2779

[22] Stevens M J, Robbins M O and Belak J F 1991 Phys. Rev. Lett. 663004

[23] Stevens M J and Robbins M O 1993 Phys. Rev. E 483778

[24] Messina R and Löwen H 2005 Relaxation back to equilibrium after cessation of shear for confined colloidal bilayers, at press

[25] Derks D, Wisman H, van Blaaderen A and Imhof A 2004 J. Phys.: Condens. Matter 16 S3917

[26] Cohen I, Mason T G and Weitz D A 2004 Phys. Rev. Lett. 93046001 